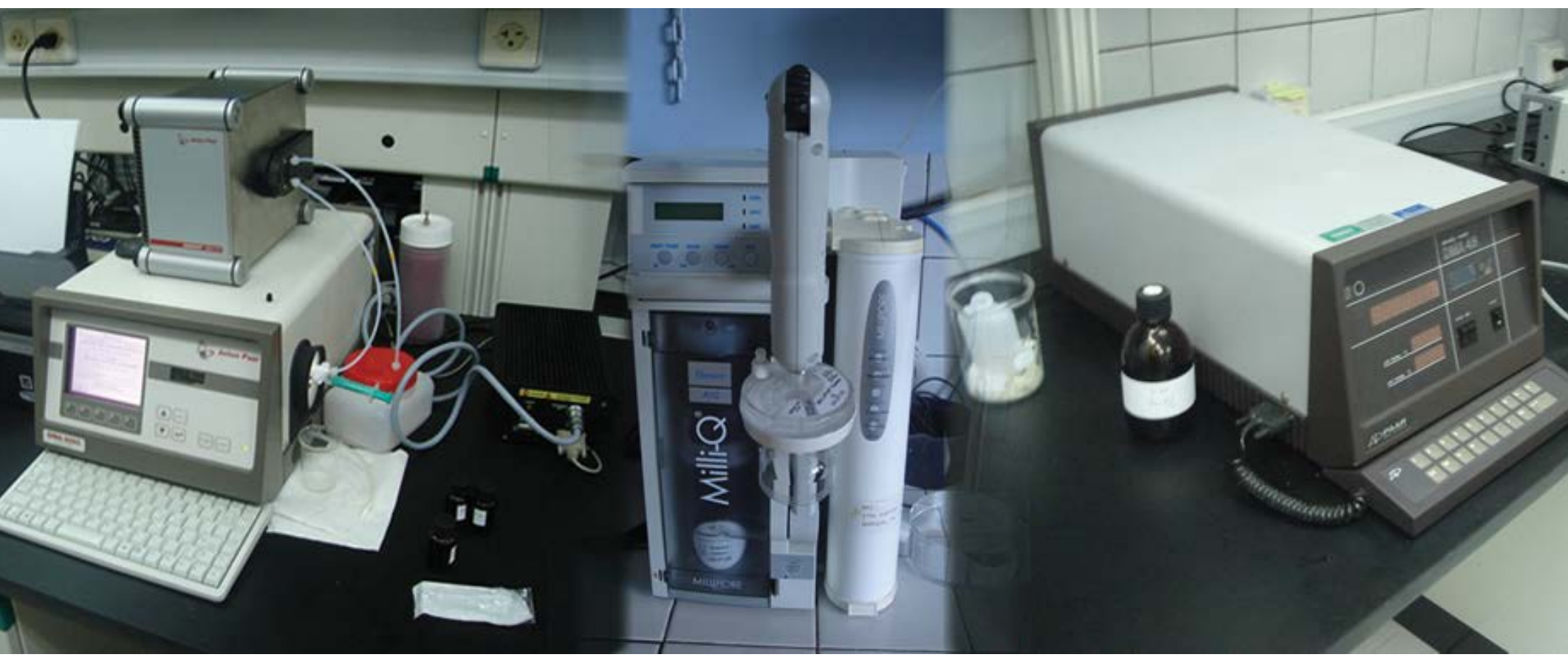

\title{
Preparación y certificación de un Material de Referencia en densidad de agua ultra pura
}

Fecha de recepción: $\quad 11$ de junio del 2012

Fecha de aceptación: 30 de julio del 2012

\section{Resumen}

La conductividad y dureza total en muestras de agua producida por cuatro combinaciones de equipos de purificación de agua fueron estudiadas. Se produjo agua ultrapura mediante diferentes métodos, eligiéndose la metodología que con que se obtuvieron muestras de agua con una conductividad y dureza total menor. Se llevaron a cabo varios procesos para la certificación de los materiales entre ellos: estudio de la conductividad en función del tiempo, el estudio del efecto de los procesos de llenado y envase, de asignación de valor de la densidad a cuatro temperaturas diferentes, utilizando métodos y variables de los estudios de estabilidad en las magnitudes de la conductividad y la densidad. Finalmente los resultados de la certificación fueron reportados en el certificado para el material de referencia certificado en densidad de los lotes de agua ultra pura.

\section{Palabras clave:}

Metrología, trazabilidad, MRC (material de referencia certificado), densidad, metrology, traceability, CRM (Certified Reference Material), density.
Humberto Mora Murillo y Jessica Chavarría Sánchez Labratorio Costarricense de Metrología (LACOMET)

E:mail: jchavarria@lacomet.go.cr

\section{Abstract}

Conductivity and total hardness in ultrapure water samples produced by four combinations of purification water equipment were studied. Production of reference material method chose the group of purification equipment which allow obtaining water samples with minor conductivity and total hardness. Several processes for the certification of the material were performed as well: study of conductivity versus time study of the effect of filling and package processes, density value assignment, four different temperatures, using variable methods and stability studies in the magnitudes of conductivity and density. Finally results of the certification were reported in the certificate for the certified reference material in density of ultra-pure water batch.

\section{Key Words:}

Metrology, traceability, CRM (Certified Reference Material), density. 


\section{Introducción}

Los procesos de calibración en metrología, permiten conocer la magnitud de los errores en los equipos o patrones de medición. Por otro lado, mediante un proceso de verificación se puede determinar el cumplimiento de requisitos de de acuerdo a parámetros conocidos.

Los Materiales de Referencia Certificados, que cumplen con lo establecido en normas internacionales para su preparación, certificación y venta, son una opción de patrones que se utilizan calibrar instrumentos, de manera que proporcione un respaldo a mediciones que estos realizan.

Según el Vocabulario Internacional de Metrología (VIM), un Material de Referencia (MR), se define como un material suficientemente homogéneo y estable, con respecto a propiedades específicas, establecido como apto para su uso previsto en una medición o en un examen de propiedades cualitativas. Un Material de Referencia Certificado (MRC), se define como un Material de Referencia que se encuentra acompañado de documentación emitida por un organismo autorizado, y proporciona uno o varios valores de propiedades especificadas, con incertidumbres y trazabilidades asociadas, empleando procedimientos válidos (1).

Dada la importancia de los MR, se han desarrollado procedimientos y métodos para que se lleve a cabo su producción de una manera sistemática, con la cual se puede garantizar la exactitud del parámetro y su trazabilidad. Las etapas para la elaboración y certificación de un MR son: (2)

-Identificación de la necesidad

-Determinación de especificaciones

-Preparación del material

- Estudio de Homogeneidad

-Asignación del valor de la propiedad de interés

- Estudio de estabilidad

-Emisión del certificado

Para la asignación del valor de la propiedad o propiedades de interés al Material de Referencia se pueden utilizar:

- Un método de medición y un método de diferente principio como confirmación

- Un método primario de medición

- Un método secundario y la participación en intercomparaciones ${ }^{(2)}$.

Para la magnitud de densidad, se pueden utilizar materiales de referencia de agua ultra pura. Este líquido debido a su abundancia, homogeneidad y estabilidad se ha estudiado como patrón de densidad durante mucho tiempo, prueba de esto es que su trazabilidad puede definirse mediante dos fuentes: Una ecuación que relaciona la densidad con la temperatura, (ecuación de Tanaka), o la calibración contra el patrón primario en densidad (esfera) ${ }^{(3)}$.

La ecuación de Tanaka se deriva de múltiples estudios realizados por diferentes investigadores y metrólogos, a lo largo del mundo, los cuales presentaron discutieron y unificaron los resultados de sus investigaciones sobre densidad y propiedades del agua media oceánica (SMOW), para obtener un modelo matemático que relaciona la densidad del agua con la temperatura de la misma. Para que una muestra de agua pueda cumplir con esta ecuación debe cumplir una serie de parámetros, dentro de los cuales los más importantes son los siguientes ${ }^{(3)}$ :

- La ecuación aplica para un intervalo de temperaturas ente $0^{\circ} \mathrm{C}$ y $40^{\circ} \mathrm{C}$ y una presión atmosférica de referencia. Si se utiliza la ecuación bajo otras condiciones de presión atmosférica se debe aplicar un factor de corrección por compresibilidad.

- Deben realizarse estudios de $\mathrm{CO}_{2}$ disuelto, para poder aplicar un factor de corrección adecuado para este parámetro. En caso de no contar con información experimental sobre la concentración de $\mathrm{CO}_{2}$ disuelto, se debe utilizar el factor de corrección para agua ultra pura con alto contenido de gases disueltos.

- La ecuación solo aplica para agua con alto grado de pureza, por esta razón se deben utilizar equipos para realizar la purificación del agua en especial se debe eliminar al máximo el contenido de sales disueltas. La pureza del agua entonces se relaciona con su valor de conductividad.

El modelo matemático presentado y publicado en el 2001, que describe el comportamiento de la densidad del agua en función de su temperatura, y que define la trazabilidad del agua ultra pura dentro de la cadena de trazabilidad en densidad, se muestra a continuación:

$$
\rho=\alpha_{5}\left[1-\frac{\left(t+\alpha_{1}\right)^{2}\left(t+\alpha_{2}\right)}{\alpha_{3}\left(t+\alpha_{4}\right)}\right]\left[1+\left(k_{0}+k_{1}+k_{2} t^{2}\right) \Delta P\right]+\left(s_{0}+s_{1} t\right)
$$

Donde (3):

$\rho=$ Densidad del agua ultra pura en $\mathrm{kg} / \mathrm{m} 3$

$a_{5}=$ Coeficiente de la ecuación. Tiene un valor de $(999,97495$

$\pm 0,00084)$ kgm-3

$\mathrm{t}=$ Temperatura del agua

$a_{1}=$ Coeficiente de la ecuación. 
$\mathrm{a}_{2}=$ Constante de la ecuación.

$a_{3}=$ Constante de la ecuación.

$a_{4}=$ Constante de la ecuación.

$\mathrm{k}_{0}, \mathrm{k}_{1}$ y $\mathrm{k}_{2}=$ Constantes del factor de corrección por compresibilidad.

$\Delta \mathrm{P}=$ Diferencia entre la presión atmosférica de referencia y la presión atmosférica medida.

$\mathrm{s}_{0}$ y $\mathrm{s}_{1}=$ Constantes del factor de corrección por gases disueltos.

El método para determinar la densidad de un líquido, utilizando un patrón sólido, consiste en determinar el cambio de masa producido al introducir el patrón sólido, generalmente una esfera, cuyo valor de volumen se conoce y cuenta con trazabilidad referenciada a un Centro Nacional de Metrología, dentro de un recipiente con la muestra líquida a la cual se le desea determinar la densidad. El recipiente y su contenido, se colocan sobre el plato de una balanza. La esfera se coloca en un soporte universal, de tal forma que no tenga contacto con el plato de la balanza, de esta forma el cambio en la masa registrado, cuando se introduce la esfera en la muestra, se debe únicamente a la masa del líquido desplazado por la esfera. Se pueden utilizar patrones de masa para corregir la lectura de la balanza.

La densidad del líquido de interés se determina aplicando la siguiente ecuación ${ }^{(4)}$ :

$$
\rho_{l 20}=\frac{\Delta m * \rho_{a} *\left(\frac{m_{c p}}{\rho_{p}}\right) *\left[1+\alpha_{p} *\left(t_{r}-t_{a}\right)\right]}{V_{s *}\left[1+\alpha_{s} *\left(t_{r}-t_{w}\right)\right]}
$$

Donde:

pl20 = Densidad del líquido a $20^{\circ} \mathrm{C}$

$\Delta \mathrm{m}=$ Masa, corregida con patrones de masa, debida al desplazamiento del liquido por la introducción de la esfera.

$\rho_{\mathrm{a}}=$ Densidad del aire. (5)

$\mathrm{m}_{\mathrm{cp}}=$ Masa Convencional patrones

$\rho_{\mathrm{p}}=$ Densidad de los patrones de masa

$\alpha_{p}=$ Coeficiente de expansión térmica de los patrones de masa

$t_{r}=$ Temperatura de referencia

$t_{a}=$ Temperatura del aire

$\mathrm{t}_{\mathrm{w}}=$ Temperatura del agua

$\alpha_{s}=$ Coeficiente de expansión térmica de la esfera

$\mathrm{V}_{\mathrm{s}}=$ Volumen de la esfera

Otro método que se puede utilizar para determinar la densidad de un líquido, a una cierta temperatura, consiste en utilizar un densímetro de oscilación; este equipo cuenta con una celda, de dimensiones conocidas, donde se inyecta la muestra líquida, el equipo puede calentar o enfriar la celda para determinar la densidad a diferentes temperaturas. Por medio de interferencias de ondas, el equipo cuenta con un algoritmo que le permite determinar rápidamente la densidad a la temperatura seleccionada. Además este tipo de equipos se calibran y controlan utilizando Materiales de Referencia Certificados en densidad.

El agua con altos niveles de pureza, o valores bajos de conductividad eléctrica, tiene muchas aplicaciones tanto en la metrología como en la química, dentro de las cuales se puede mencionar:

- Preparación de disoluciones patrón para usos en: curvas de regresión, validar metodologías, realizar estudios estadísticos o controles de calidad.

- Preparar disoluciones salinas que se utilizan en la calibración de patrones de humedad relativa.

-Preparación de la celda del punto triple del agua, que es el patrón primario en esta magnitud.

- Se utiliza como patrón de densidad en calibraciones volumétricas por el método gravimétrico

-Preparación de MRC en varias áreas, dentro de la que se pueden citar densidad, conductividad y $\mathrm{pH}$.

Debido a la gran variedad de aplicaciones que tiene el agua de alta pureza dentro de la metrología y la química, además de la necesidad de contar con MRC en densidad para la calibración y controles de equipos en Costa Rica, es que se realiza la preparación y certificación de un Material de Referencia de agua ultra pura. Este proceso se realizó en el Laboratorio Costarricense de Metrología (LACOMET).

\section{Sección experimental y resultados}

\subsection{Selección del Conjunto de equipos para purificación del agua potable}

Cuadro 1. Equipos

\begin{tabular}{|c|c|c|}
\hline Conjunto equipos & Equipos & Ubicación LACOMET \\
\hline 1 & $\begin{array}{c}\text { Filtro carbono-UV, filtro } \\
\text { de pretratamiento, } \\
\text { destilador }\end{array}$ & Laboratorio de Volumen \\
\hline 2 & $\begin{array}{c}\text { Filtro carbono-UV, filtro } \\
\text { de pretratamiento, } \\
\text { bidestilador }\end{array}$ & Laboratorio de Volumen \\
\hline 3 & $\begin{array}{c}\text { Filtro carbono-UV, filtro } \\
\text { de pretratamiento, } \\
\text { bidestilador, } \\
\text { desionizador }\end{array}$ & $\begin{array}{c}\text { Volumen, Metrología } \\
\text { Química }\end{array}$ \\
\hline 4 & $\begin{array}{r}\text { Filtro carbono-UV, } \\
\text { filtro de sedimentos, } \\
\text { destilador, desionizador }\end{array}$ & $\begin{array}{c}\text { Laboratorio de } \\
\text { Metrología Química }\end{array}$ \\
\hline
\end{tabular}


En el cuadro II se muestran los resultados promedio de la medición de conductividad, que se realizaron con un conductímetro calibrado, y de dureza total, obtenidos por valoración con ácido etilendiamintetracético (EDTA), para las muestras de agua ultra pura producidas por cada uno de las combinaciones de equipos del cuadro I.

Para las muestras obtenidas por el conjunto de equipos de purificación 3 y 4 no fue posible cuantificar la dureza total, ya los valores estaban por debajo del límite de detección de la metodología de medición.

Cuadro 2. Resultados promedio de conductividad y dureza total para muestras de agua ultra pura

\begin{tabular}{|c|c|c|}
\hline Conjunto equipos & Conductividad $\mu \mathrm{S} / \mathrm{cm}$ & Dureza total $\mathrm{mg} / \mathrm{L}$ \\
\hline 1 & $4,58 \pm 0,95$ & $12 \pm 0,9$ \\
\hline 2 & $1,31 \pm 0,57$ & $1,9 \pm 1,7$ \\
\hline 3 & $0,76 \pm 0,52$ & ---- \\
\hline 4 & $1,19 \pm 0,52$ & ---- \\
\hline
\end{tabular}

Según los resultados del cuadro II, el conjunto de equipos elegidos para la preparación del Material de Referencia fue el número 3 , ver cuadro I, debido a que presentaron los menores valores de conductividad y dureza total.

Una vez seleccionado el conjunto de equipos, se procedió a realizar un estudio de conductividad en el tiempo del agua ultra pura producida por los mismos, para garantizar que se mantienen los niveles de pureza. En el cuadro III se muestran los resultados promedio obtenidos en este estudio.

Cuadro 3. Resultados promedio de conductividad para muestras de agua ultra pura producidas en diferentes intervalos de tiempo

\begin{tabular}{|c|c|c|}
\hline Mes de Producción & $\begin{array}{c}\text { Conductividad medida } \\
\mu \mathrm{S} / \mathrm{cm}\end{array}$ & Temperatura ${ }^{\circ} \mathrm{C}$ \\
\hline 1 & $0,71 \pm 0,50$ & 24,8 \\
\hline 2 & $0,84 \pm 0,50$ & 25,2 \\
\hline 4 & $0,99 \pm 0,50$ & 24,6 \\
\hline 5 & $0,89 \pm 0,50$ & 24,6 \\
\hline 7 & $0,88 \pm 0,50$ & 25,5 \\
\hline 8 & $0,72 \pm 0,50$ & 25,5 \\
\hline 10 & $0,83 \pm 0,50$ & 25,1 \\
\hline
\end{tabular}

De los resultados anteriores se puede apreciar que el valor de conductividad, tomando en cuenta la incertidumbre del equipo de medición, se mantuvo estable en un largo período de tiempo, dentro del cual se realizaron los mantenimientos y cambios de cartuchos de los equipos de purificación del agua recomendados por el fabricante. Con este estudio se puede garantizar que el nivel de pureza del material producido se mantiene a través del tiempo evaluado.

Con los estudios de estabilidad del proceso de producción del Material de Referencia se procedió a producir un lote de aproximadamente $15 \mathrm{~L}$ de agua ultra pura. Mediante la aplicación de técnicas estadísticas de muestreo (5), se tomaron y envasaron, en frascos ámbar de aproximadamente $100 \mathrm{~cm} 3$, muestras del lote de agua ultra pura controlando la velocidad y hora de llenado y el nivel volumétrico de envasado. Luego se tomaron otras muestras del lote sin controlar ninguno de estos factores y así poder estudiar el efecto de llenado sobre las propiedades de interés del Material de Referencia.

En el cuadro IV, se presentan los resultados obtenidos al determinar la conductividad y la densidad a $20^{\circ} \mathrm{C}$ promedio de cada conjunto de muestras.

Cuadro 4. Resultados de conductividad a $25^{\circ} \mathrm{C}$ y de densidad a $20^{\circ} \mathrm{C}$ promedio para cada conjunto de muestras en el estudio de efecto de encasado

\begin{tabular}{|c|c|c|}
\hline Mes de Producción & $\begin{array}{c}\text { Conductividad medida } \\
\mu \mathrm{S} / \mathrm{cm}\end{array}$ & Temperatura ${ }^{\circ} \mathrm{C}$ \\
\hline 1 & $0,71 \pm 0,50$ & 24,8 \\
\hline 2 & $0,84 \pm 0,50$ & 25,2 \\
\hline 4 & $0,99 \pm 0,50$ & 24,6 \\
\hline 5 & $0,89 \pm 0,50$ & 24,6 \\
\hline 7 & $0,88 \pm 0,50$ & 25,5 \\
\hline 8 & $0,72 \pm 0,50$ & 25,5 \\
\hline 10 & $0,83 \pm 0,50$ & 25,1 \\
\hline
\end{tabular}

La t crítica al $95 \%$ de confianza para ambos casos fue de 2,179

Con una prueba $\mathrm{t}$ de Student, para evaluar si existe diferencia entre los valores de conductividad y densidad medidos a muestras de agua ultra pura con controles de llenado y muestras de agua ultra pura sin controlar ningún factor en el llenado, se pudo comprobar que la diferencia no es significativa al $95 \%$ de confianza. Lo anterior indica que no existe influencia en el valor del material debida al efecto de llenado o envasado en las propiedades de interés del Material de Referencia.

Posteriormente se procedió a la asignación del valor de referencia de la densidad. Para este proceso se utilizaron tres metodologías de diferente principio, para la asignación del valor de densidad a cuatro temperaturas diferentes: 
$15,5{ }^{\circ} \mathrm{C}, 20{ }^{\circ} \mathrm{C}, 22{ }^{\circ} \mathrm{C}$ y $25^{\circ} \mathrm{C}$. La primera metodología empleada fue la ecuación de Tanaka, y se utilizaron como metodologías de confirmación el método de pesada hidrostática, utilizando un patrón sólido de densidad, y el método de medición directa utilizando un densímetro de oscilación. Es importante destacar que se utilizó la ecuación de Tanaka aplicando los factores de corrección por gases disueltos y por compresibilidad, además se comprobó con los estudios de conductividad que el agua ultra pura cumple con los requisitos de pureza para poder aplicar dicha ecuación. En el cuadro V, se muestran los resultados. Para las confirmación del valor y el estudio de estabilidad a tres diferentes tiempos, se tomaron cuarenta muestras de aproximadamente $100 \mathrm{~cm} 3$, en sus respectivos frascos ámbar, del Lote de agua ultra pura. Se aplicó un método matricial para seleccionar diez muestras para cada proceso.

Cuadro 5. Resultados obtenidos al asignar el valor de densidad al Material de Referencia aplicando la ecuación de Tanaka

\begin{tabular}{|c|c|c|}
\hline Temperatura & Densidad $\mathrm{g} / \mathrm{cm}_{3}$ & $\mathrm{U}(\mathrm{k}=2) \mathrm{g} / \mathrm{cm}_{3}$ \\
\hline 1 & 0,99902 & 0,00003 \\
\hline 2 & 0,99820 & 0,00004 \\
\hline 3 & 0,99776 & 0,00004 \\
\hline 4 & 0,99704 & 0,00005 \\
\hline
\end{tabular}

En las figura 1 se muestra el gráfico donde se comparan los valores de densidad asignados utilizando la ecuación de Tanaka y los obtenidos con un patrón sólido de densidad y por medición con el densímetro de oscilación.

Los valores de densidad obtenidos por diferentes metodologías no presentan diferencias significativas entre sí, esto además se corroboró con una prueba de error normalizado. Todos los errores normalizados fueron menores a 1, excepto para la primer temperatura donde se obtuvo una diferencia significativa, la cual se debe a que el densímetro de oscilación utilizado, fue calibrado con materiales de referencia certificados en densidad, a temperaturas superiores a la temperatura 1 , por lo tanto se presenta una desviación en esta temperatura que no es corregida por la calibración y esto deriva en una diferencia respecto al valor asignado por la ecuación de Tanaka que no se presenta en los valores de densidad de las demás temperaturas estudiadas.

Para el estudio de estabilidad se almacenaron las treinta muestras por diferentes períodos de tiempo: quince días, un mes y dos meses. Se realizaron pruebas de conductividad a $25^{\circ} \mathrm{C}$, para demostrar el cumplimiento con las condiciones para aplicar la ecuación de Tanaka, y pruebas de densidad a una de las temperaturas estudiadas, utilizando el densímetro de oscilación. Lo anterior se aplicó a diez muestras, seleccionadas por el método de la matriz, al cumplirse cada ciclo de tiempo estudiado.

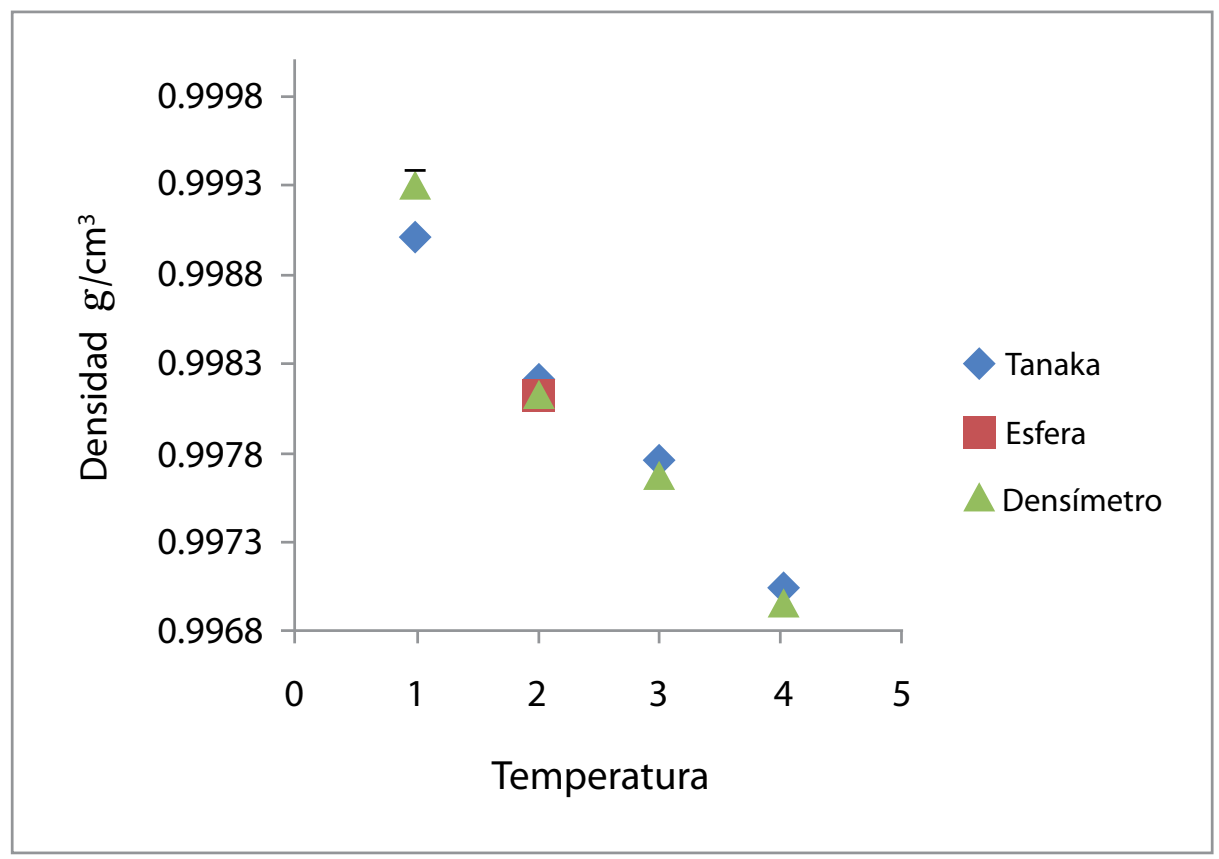

Figura 1. Gráfico comparativo de valores de densidad a cuatro diferentes temperaturas, obtenidos aplicando diferentes metodologías 


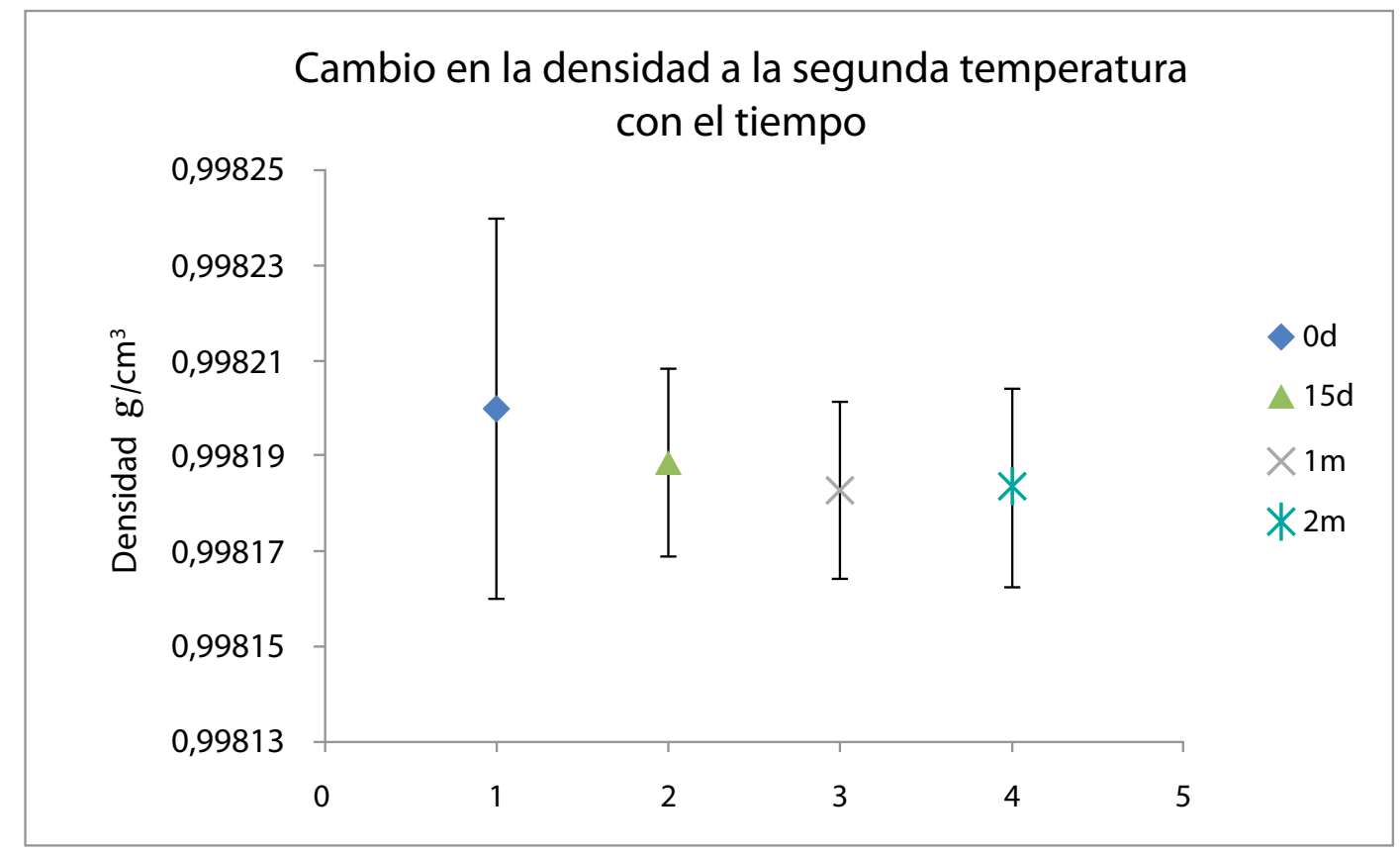

Figura 2. Comparación de los valores de densidad, en el tiempo, medidos con el densímetro de oscilación, contra el valor asignado por la ecuación de Tanaka, a la segunda temperatura

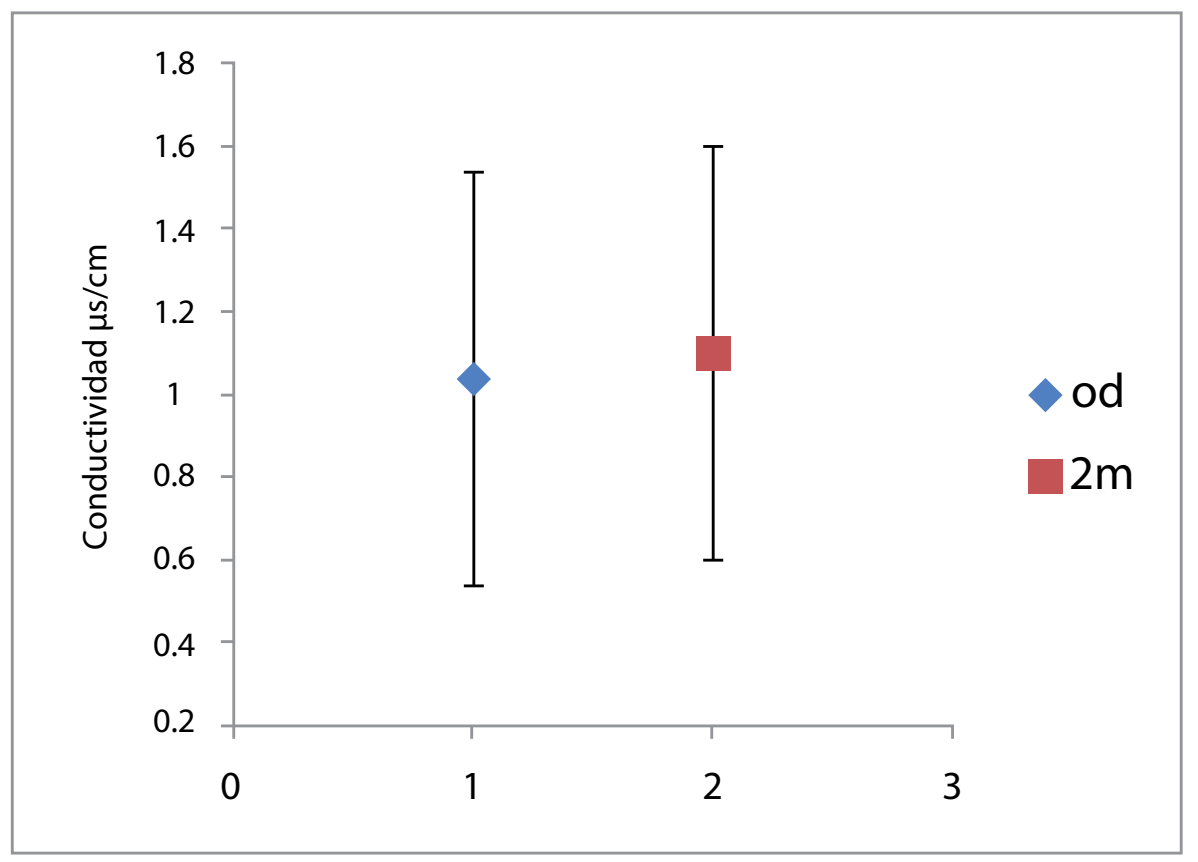

Figura 3. Comparación de valor de conductividad medida previo a la asignación del valor de densidad contra el valor de conductividad medida a los dos meses de almacenar el Material de Referencia

En las figuras 2 y 3 se aprecian los resultados para el estudio de estabilidad en densidad y conductividad.

De los resultados anteriores se desprende que no existe diferencia significativa tanto en los valores de densidad como en los valores de conductividad, luego del período de almacenamiento, esto se corroboró aplicando una prueba de error normalizado. 
Finalmente se elabora el certificado del Material de Referencia contemplando lo establecido en la Guía ISO 31:2000. Contenido de las etiquetas y Certificados de los Materiales de Referencia. Además todos los cálculos se realizaron utilizando hojas de cálculo de Excel, las cuales fueron validadas utilizando el software GUM Workbench.

\section{Conclusiones}

Se realizaron estudios de desempeño de diferentes equipos y combinaciones de los mismos para la purificación del agua potable. Se demostró que el agregar un desionizador a un proceso de filtración y bidestilación de agua, permite obtener una mejor calidad de agua ultra pura en lo que a dureza total y conductividad eléctrica se refiere.

Se estableció, mediante estudios, que el agua ultra pura producida por el conjunto de equipos seleccionados, cumple con los principales requisitos establecidos para poder aplicar la ecuación de Tanaka, sin embargo para poder declarar total cumplimiento de requisitos, se requieren estudios de composición isotópica y gases disueltos en el agua ultra pura. Se demostró, con un historial de conductividad del agua ultra pura, que el conjunto de equipos seleccionados para producir el MRC no influyen significativamente en la pureza del mismo.

Se comprobó que la propiedad de interés en el material producido, es suficientemente homogénea ya que no hay evidencia de influencia significativa del proceso de llenado y envasado sobre la propiedad de interés del MRC.

Con las condiciones de calibración y características metrológicas de todos los equipos utilizados, y con el mantenimiento apropiado de los equipos para la purificación del agua, se logró obtener un MRC con un grado de exactitud e incertidumbre adecuados para la calibración y controles metrológicos de densímetros digitales a nivel industrial. Este MRC también permite realizar controles metrológicos a los conductímetros.

Como se demostró experimentalmente con las pruebas de estabilidad, las propiedades certificadas al MR son lo suficientemente estables, al menos durante un período de dos meses.

Al realizarse la producción y asignación del valor de este MRC en el LACOMET, el producto puede ser ofrecido a precios significativamente menores que los encontrados a nivel internacional. Comprar un MR en el extranjero implica, a parte de su alto precio, un costo extra por traslados y manejos aduanales para que el producto pueda entrar a Costa Rica, esto no sucede con el MRC en densidad producido.

Este estudio no solamente le permite contar a los laboratorios de metrología con patrones trazables, para realizar calibraciones en la magnitud de densidad, sino que también les permite a los laboratorios de ensayo o calibración, realizar controles metrológicos, o controles intermedios, a sus equipos, en lo que respecta a conductividad y densidad, de manera que pueden cumplir con lo establecido en la norma INTE/IESO-IEC 17025.

\section{Referencias}

1. BIPM/IEC/IFF/ILAC/ISO/IUPAP/OIML. Vocabulario Internacional de Metrología-Conceptos Fundamentales y Generales, y Términos Asociados (VIM). Traducción del VIM 3ra Edición. Marzo 2009.

2. Organización Internacional de Normalización (ISO). Guía 34:2000. Requerimientos Generales para la competencia de productores de Materiales de Referencia.

3. Organización Internacional de Normalización (ISO). Guía 30:1992. Términos y Definiciones utilizados en relación a los Materiales de Referencia.

4. Tanaka, M; Girad, G; Davis, R; Peuto, A; Bignell, N. Recommended table for the density of water between $O C$ and $40 \mathrm{C}$ based on recent experimental reports. Metrología. 2001.

5. Sartorius. Manual of Weighing Applications Part 1 Density. Alemania. Febrero 1999.

6. Reglamento Técnico Centroamericano numero RTCA 01.01.11:06 Cantidad de Producto en Preempacados. Ministerio de Comercio Exterior y Ministerio de Economía Industria y Comercio. 2006

7. Becerra, L.O. Guardado, M.E. Estimación de la Incertidumbre en la Determinación de la Densidad del Aire. Centro Nacional de Metrología (CENAM), 2003. pp 3.

8. Becerra. L.O; Daued. A; Muñoz. F. Guía Técnica para la Calibración de Densímetros de Funcionamiento Electrónico de Indicación Digital. Centro Nacional de Metrología (CENAM)/ Entidad Mexicana de Acreditación (EMA). México. Enero 2005

9. Organización Internacional de Normalización (ISO). Guía 35:2006. Materiales de Referencia, Principios Generales y Estadísticos para certificación.

10. Organización Internacional de Normalización (ISO). Guía 33:2000. Uso de los Certificados de Materiales de Referencia.

11. Organización Internacional de Normalización (ISO). Guía 34:2000. Requerimientos Generales para la competencia de productores de Materiales de Referencia. 